\title{
Transcatheter Aortic-Valve Replacement for Inoperable Severe Aortic Stenosis
}

\author{
Raj R. Makkar, M.D., Gregory P. Fontana, M.D., Hasan Jilaihawi, M.D., \\ Samir Kapadia, M.D., Augusto D. Pichard, M.D., Pamela S. Douglas, M.D., \\ Vinod H. Thourani, M.D., Vasilis C. Babaliaros, M.D., John G. Webb, M.D., \\ Howard C. Herrmann, M.D., Joseph E. Bavaria, M.D., Susheel Kodali, M.D., \\ David L. Brown, M.D., Bruce Bowers, M.D., Todd M. Dewey, M.D., \\ Lars G. Svensson, M.D., Ph.D., Murat Tuzcu, M.D., Jeffrey W. Moses, M.D., \\ Matthew R. Williams, M.D., Robert J. Siegel, M.D., Jodi J. Akin, M.S., \\ William N. Anderson, Ph.D., Stuart Pocock, Ph.D., Craig R. Smith, M.D., \\ and Martin B. Leon, M.D., for the PARTNER Trial Investigators*
}

\section{A B STRACT}

From Cedars-Sinai Heart Institute, Los Angeles (R.R.M., H.J., R.J.S.); Lenox Hill Heart and Vascular Institute (G.P.F.) and Columbia University Medical Center and New York Presbyterian Hospital (S. Kodali, J.W.M., M.R.W., C.R.S., M.B.L.) both in New York; Cleveland Clinic Foundation, Cleveland (S. Kapadia, L.G.S., M.T.); Washington Hospital Center, Washington, DC (A.D.P.); Duke University School of Medicine, Durham, NC (P.S.D.); Emory University School of Medicine, Atlanta (V.H.T., V.C.B.), University of British Columbia and St. Paul's Hospital, Vancouver, Canada (J.G.W.); Hospital of the University of Pennsylvania, Philadelphia (H.C.H., J.E.B.); Baylor Healthcare System (D.L.B., B.B.) and Medical City Dallas (T.M.D.) - both in Dallas; Edwards Lifesciences, Irvine, CA (J.J.A., W.N.A.); and London School of Hygiene and Tropical Medicine, London (S.P.). Address reprint requests to Dr. Leon at Columbia University Medical Center, Center for Interventional Vascular Therapy, 161 Fort Washington Ave., 6th Fl., New York, NY 10032, or at mleon@crf.org.

*The investigators, institutions, and research organizations participating in the Placement of Aortic Transcatheter Valves (PARTNERS) trial are listed in the Supplementary Appendix, available at NEJM .org.

This article (10.1056/NEJMoal202277) was published on March 26, 2012, and updated on August 30, 2012, at NEJM.org.

N Engl J Med 2012;366:1696-704.

Copyright $\odot 2012$ Massachusetts Medical Society.

\section{BACKGROUND}

Transcatheter aortic-valve replacement (TAVR) is the recommended therapy for patients with severe aortic stenosis who are not suitable candidates for surgery. The outcomes beyond 1 year in such patients are not known.

\section{METHODS}

We randomly assigned patients to transfemoral TAVR or to standard therapy (which often included balloon aortic valvuloplasty). Data on 2-year outcomes were analyzed. RESULTS

A total of 358 patients underwent randomization at 21 centers. The rates of death at 2 years were $43.3 \%$ in the TAVR group and $68.0 \%$ in the standard-therapy group $(\mathrm{P}<0.001)$, and the corresponding rates of cardiac death were $31.0 \%$ and $62.4 \%$ $(\mathrm{P}<0.001)$. The survival advantage associated with TAVR that was seen at 1 year remained significant among patients who survived beyond the first year (hazard ratio, $0.58 ; 95 \%$ confidence interval [CI], 0.36 to $0.92 ; \mathrm{P}=0.02$ with the use of the log-rank test). The rate of stroke was higher after TAVR than with standard therapy $(13.8 \%$ vs. $5.5 \%, \mathrm{P}=0.01$ ), owing, in the first 30 days, to the occurrence of more ischemic events in the TAVR group ( $6.7 \%$ vs. $1.7 \%, \mathrm{P}=0.02)$ and, beyond 30 days, to the occurrence of more hemorrhagic strokes in the TAVR group $(2.2 \%$ vs. $0.6 \%, P=0.16)$. At 2 years, the rate of rehospitalization was $35.0 \%$ in the TAVR group and $72.5 \%$ in the standard-therapy group $(\mathrm{P}<0.001)$. TAVR, as compared with standard therapy, was also associated with improved functional status $(\mathrm{P}<0.001)$. The data suggest that the mortality benefit after TAVR may be limited to patients who do not have extensive coexisting conditions. Echocardiographic analysis showed a sustained increase in aortic-valve area and a decrease in aortic-valve gradient, with no worsening of paravalvular aortic regurgitation.

\section{CONCLUSIONS}

Among appropriately selected patients with severe aortic stenosis who were not suitable candidates for surgery, TAVR reduced the rates of death and hospitalization, with a decrease in symptoms and an improvement in valve hemodynamics that were sustained at 2 years of follow-up. The presence of extensive coexisting conditions may attenuate the survival benefit of TAVR. (Funded by Edwards Lifesciences; ClinicalTrials.gov number, NCT00530894.) 
YMPTOMATIC AORTIC STENOSIS, IF LEFT untreated, is characterized by a high risk of death. ${ }^{1-6}$ In the randomized Placement of Aortic Transcatheter Valves (PARTNER) trial, transcatheter aortic-valve replacement (TAVR), as compared with standard therapy, in patients who were not considered to be suitable candidates for surgery, decreased the rate of death at 1 year, reduced cardiac symptoms, and improved the hemodynamic performance of the valve. ${ }^{2}$ Longer-term outcomes are essential to guide clinical practice decisions in this elderly patient population, in which many of the patients have multiple coexisting conditions. Moreover, there is a paucity of long-term data on the performance of the valve, and most of the data that are available include echocardiograms that have not been analyzed at an independent core laboratory. ${ }^{7-10}$ The objective of this analysis was to report 2-year findings from the PARTNER trial and to perform subgroup analyses in order to gain a better understanding of the effect of coexisting conditions on survival.

\section{METHODS}

\section{STUDY DESIGN AND PATIENTS}

The PARTNER trial was a multicenter, randomized study involving patients with severe aortic stenosis (aortic-valve area of $<0.8 \mathrm{~cm}^{2}$ ) and with cardiac symptoms (New York Heart Association [NYHA] functional class II or higher), who were considered not to be suitable candidates for surgery because of either clinical or anatomical factors. ${ }^{2}$ The entry criteria were stringent, requiring attestation by two cardiac surgeons and an interventional cardiologist that the patient was not a suitable candidate for surgery (as indicated by a $\geq 50 \%$ probability that the patient would either die or have serious irreversible complications after conventional surgical aortic-valve replacement). Written informed consent was obtained from all the patients in the study.

Full details of the inclusion and exclusion criteria have been reported previously. ${ }^{2}$ Patients were randomly assigned, in a 1:1 ratio, to TAVR or standard therapy, which included balloon aortic valvuloplasty, at the discretion of the treating physicians.

\section{STUDY OVERSIGHT}

The trial was designed by the sponsor (Edwards Lifesciences) and members of the executive com- mittee. The sponsor participated in the selection and management of the sites and the collection and monitoring of the data. The first author, the coprincipal academic investigators, and the executive committee had unrestricted access to the data after the database was locked, made the decision to submit the manuscript for publication, prepared all drafts of the manuscript, and attest to the integrity of the trial and the completeness and accuracy of the reported data, as well as to the fidelity of the study to the trial protocol. The protocol, including the statistical analysis plan, is available with the full text of this article at NEJM.org.

All serious adverse events were adjudicated by an independent clinical events committee. All data were analyzed by a biostatistician who was paid by the sponsor and by an academic biostatistician. An independent core laboratory analyzed all echocardiograms.

\section{PROCEDURE}

The SAPIEN heart-valve system (Edwards Lifesciences), which was used in this study, consisted of a balloon-expandable, stainless-steel stent frame housing a tri-leaflet bovine pericardial valve within a deflectable delivery catheter. The system was inserted into the common femoral artery under aseptic conditions, with the use of both transesophageal and fluoroscopic guidance, while the patient was under general anesthesia. ${ }^{2}$

\section{END POINTS}

The prespecified primary end point of the PARTNER trial was all-cause mortality, over the duration of the trial. The key end points for this analysis were the 2-year rates of death from any cause, death from cardiac causes, stroke, and repeat hospitalization; NYHA functional class; number of days alive and out of the hospital; and echocardiographic assessments of aortic-valve gradients, aortic-valve area, and paravalvular regurgitation. We used the Society of Thoracic Surgeons (STS) risk score (which ranges from $0 \%$ to $100 \%$, with higher scores indicating greater surgical risk) as a measure of coexisting conditions and stratified mortality according to STS risk categories ( $<5 \%, 5$ to $14.9 \%$, and $\geq 15 \%$ ).

\section{STATISTICAL ANALYSIS}

We estimated the sample size on the basis of the primary end point (all-cause mortality). ${ }^{2}$ The analyses of clinical outcomes were performed on data 
from the intention-to-treat population, which included all patients who underwent randomization, regardless of the treatment received. Echocardiographic analyses were performed according to the treatment received. Categorical variables were compared with the use of Fisher's exact test. Continuous variables, which are presented as means with standard deviations, were compared with the use of Student's t-test. Survival curves for time-toevent variables were constructed on the basis of all available follow-up data with the use of KaplanMeier estimates and were compared by means of the log-rank test. The last date a patient was alive was derived from the database, with the use of information on office visits, telephone calls, dates on which adverse events were reported, and the actual reported date of death. A two-sided alpha level of 0.05 was used for superiority testing. All patients were followed for at least 2 years after randomization. A generalized linear model was used to calculate risk ratios in prespecified subgroups and to test for interactions. To assess the effect of balloon aortic valvuloplasty on mortality in the standard-therapy group, we performed timedependent covariate analyses. After completion of 1 year of follow-up, patients in the standardtherapy group were permitted to cross over to the TAVR group. Data from the small minority of the patients in the standard-therapy group who crossed over were censored at the time of crossover. All statistical analyses were performed with the use of SAS software, version 9.2 (SAS Institute).

\section{RESULTS}

\section{PATIENTS AND ENROLLMENT}

Patients were enrolled at 21 centers worldwide (17 in the United States) between May 11, 2007, and March 16, 2009. A total of 358 patients with severe aortic stenosis who were considered to be unsuitable candidates for surgery were assigned to either TAVR or standard therapy (Fig. 1 in the Supplementary Appendix, available at NEJM.org).

The patients enrolled in the study were elderly (mean age, 83 years) and had severe cardiac symptoms ( $93 \%$ had NYHA class III or IV symptoms). The baseline characteristics of the patients in the two groups have been described previously ${ }^{2}$ and were generally well balanced between the groups (Table 3 in the Supplementary Appendix), with the exception of the presence of chronic obstructive pulmonary disease (COPD) and the presence of atrial fibrillation, for which the rates were higher in the standard-therapy group than in the TAVR group, and the presence of extensively calcified (porcelain) aorta, for which the rate was higher in the TAVR group. Although the overall risk of surgery was high (STS score, 11.6 $\pm 6.0 \%$ ), many patients had a low STS score but had specific anatomical or clinical factors that contributed to the surgeons' assessment that they were not suitable candidates for surgery. These factors included a porcelain aorta (15.1\%), chest-wall deformity or deleterious effects of prior chest-wall irradiation (13.1\%), COPD requiring supplemental oxygen (23.5\%), and frailty, as defined according to prespecified criteria (23.1\%).

\section{TWO-YEAR CLINICAL OUTCOMES \\ Mortality}

At 2 years (Fig. 1), the rate of death from any cause was $68.0 \%$ among patients in the standardtherapy group, as compared with $43.3 \%$ among patients in the TAVR group $(\mathrm{P}<0.001)$. The outcomes were essentially unchanged when data from patients who had crossed over from standard therapy to TAVR were not censored $67.6 \%$ in the standard-therapy group vs. $43.3 \%$ in the TAVR group, $\mathrm{P}<0.001)$. The rate of death from cardiac causes was also significantly higher in the standard-therapy group than in the TAVR group (62.4\% vs. $31.0 \%, \mathrm{P}<0.001)$. The differences in survival remained significant after the first year: when the two groups were compared with the use of the proportional-hazards algorithm, conditional on survival to 1 year, the rate of death in year 2 was $18.2 \%$ in the TAVR group as compared with $35.1 \%$ in the standard-therapy group (hazard ratio with TAVR, $0.58 ; 95 \%$ confidence interval [CI], 0.36 to $0.92 ; \mathrm{P}=0.02$ with the use of the log-rank test). In a similar analysis, the rate of cardiac death in year 2 was $13.2 \%$ in the TAVR group and 32.1\% in the standard-therapy group (hazard ratio, 0.48; $95 \% \mathrm{CI}, 0.29$ to $0.81 ; \mathrm{P}=0.004$ with the use of the log-rank test).

A total of 20 of the 58 eligible patients who were alive at the time crossover was permitted (11 of whom crossed over between year 1 and year 2) were treated with TAVR, with the procedure performed an average of $699 \pm 166$ days (range, 462 to 979) after the beginning of the study. The 1-year mortality among patients who crossed over to TAVR was $10 \%$, as compared with $21 \%$ among patients who were eligible but did not cross over. 


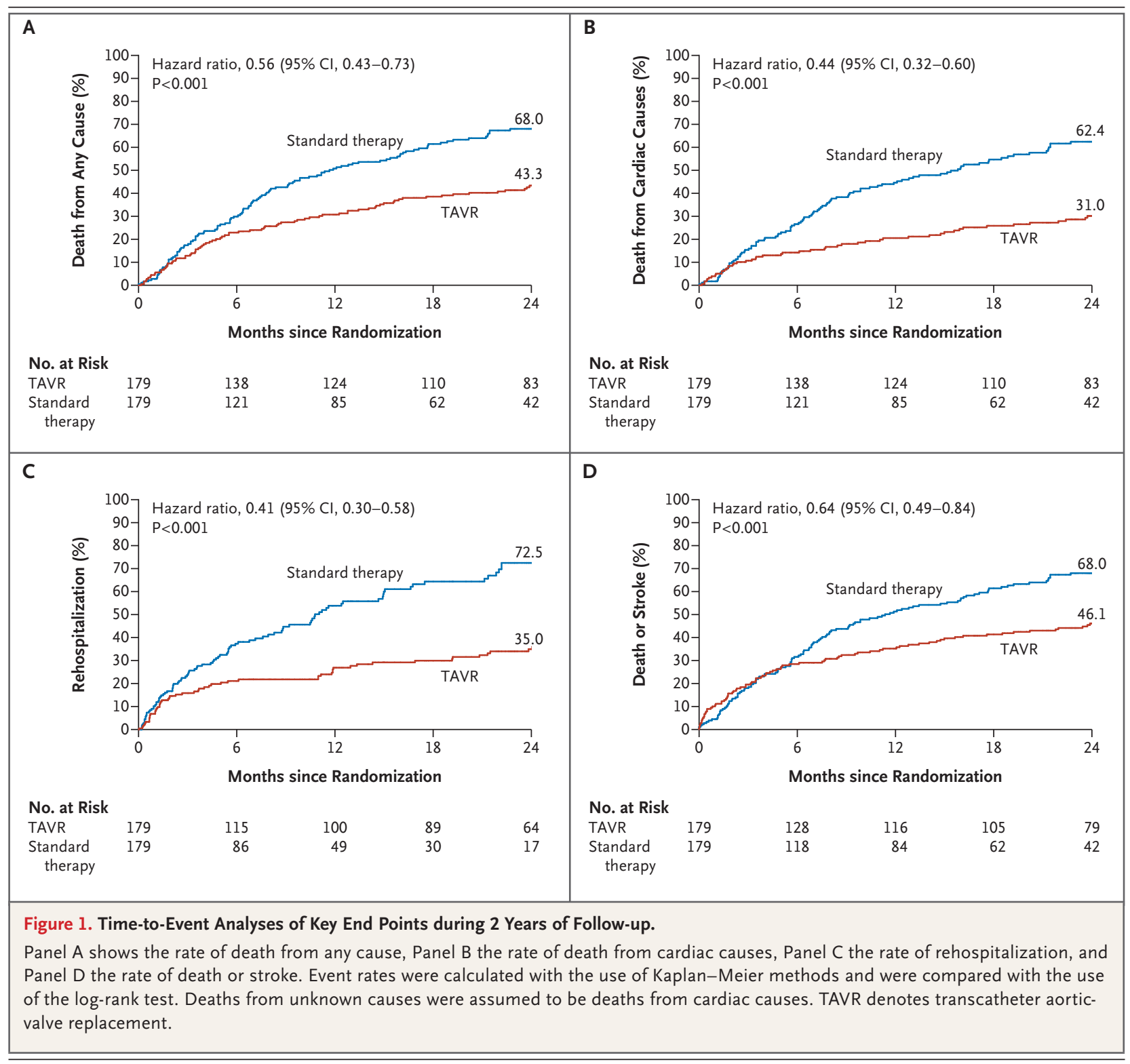

A large percentage of patients in the standardtherapy group (82.3\%) underwent balloon aortic valvuloplasty. Patients who underwent balloon aortic valvuloplasty, as compared with those who did not undergo that procedure, appeared to have favorable outcomes, at least for the first 6 months after random assignment to standard therapy (Fig. 2 in the Supplementary Appendix). However, a time-dependent covariate analysis revealed no significant difference in the outcome according to whether patients underwent balloon aortic valvuloplasty (hazard ratio with balloon aortic valvuloplasty, $1.17 ; 95 \% \mathrm{CI}, 0.75$ to $1.83 ; \mathrm{P}=0.48$ ).
Stratification of 2-year mortality according to STS score ( $<5 \%, 5$ to $14.9 \%$, or $\geq 15 \%$ ) revealed a significant association between the outcomes of TAVR and the STS score (Fig. 2), with the survival benefit of TAVR diminishing with a higher STS score ( $\mathrm{P}=0.01$ with the use of the log-rank test); however, there was no significant association between the STS score and the poor outcomes of standard therapy $(\mathrm{P}=0.67$ with the use of the log-rank test). In a multivariable proportional-hazards model for the predictors of death at 2 years among patients randomly assigned to TAVR, the only significant factors were body-mass 


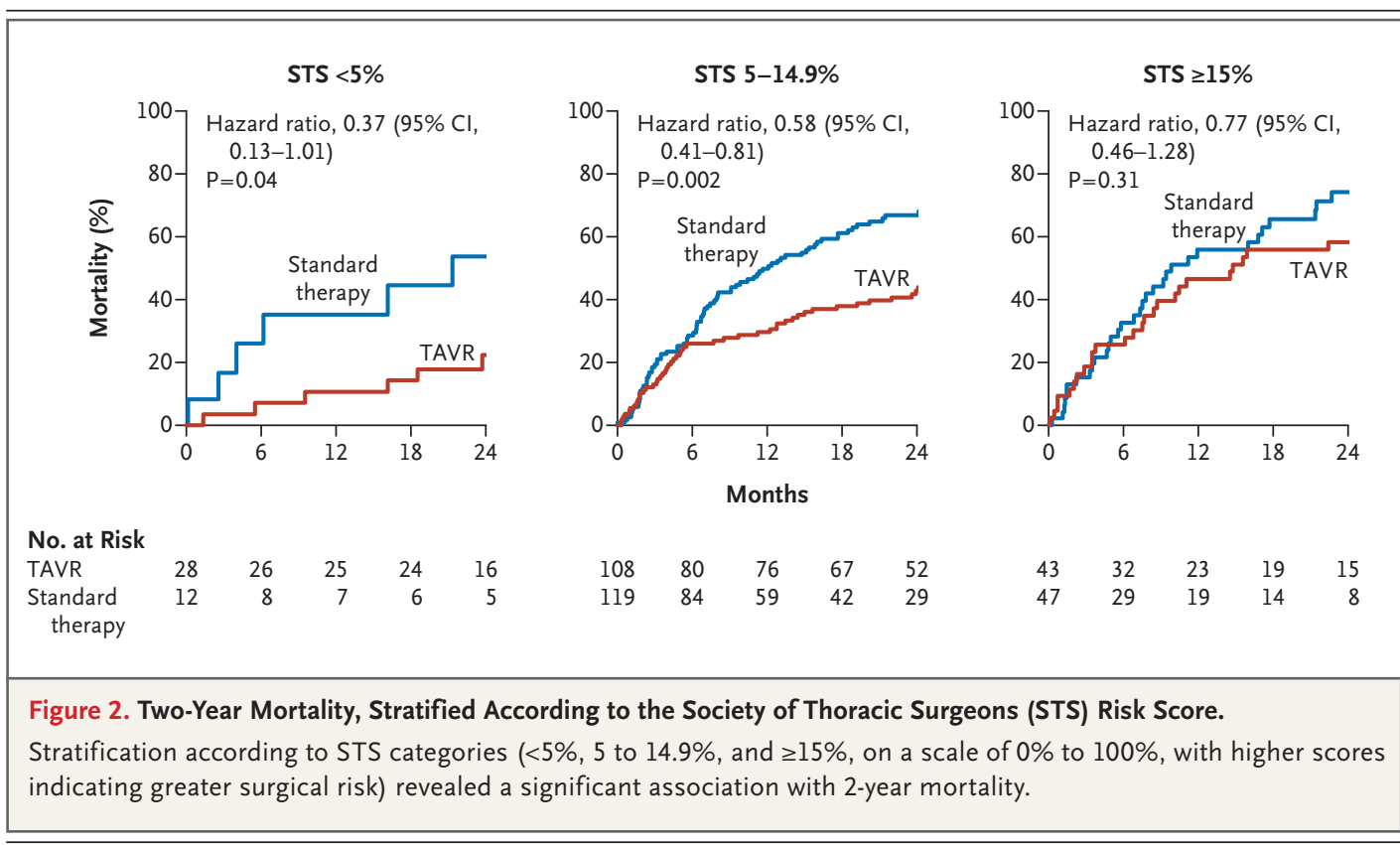

index (hazard ratio per unit increase, 0.95; 95\% CI, 0.91 to $0.98 ; \mathrm{P}=0.005$ ), prior stroke (hazard ratio, 2.99; 95\% CI, 1.19 to 7.51; $\mathrm{P}=0.01$ ), and COPD requiring supplemental oxygen (hazard ratio, 1.69; $95 \%$ CI, 1.05 to $2.73 ; \mathrm{P}=0.03$ ).

\section{Functional Status}

Among survivors at 2 years of follow-up, 83.1\% in the TAVR cohort, as compared with $42.5 \%$ in the standard-therapy cohort, were asymptomatic or had mild symptoms (NYHA class I or II symptoms) $(\mathrm{P}<0.001)$ (Fig. 3 in the Supplementary Appendix). The rate of repeat hospitalization for cardiac reasons was also lower after TAVR than with standard therapy $(35.0 \%$ vs. $72.5 \%, \mathrm{P}<0.001)$. At 2 years, the median number of days alive and out of the hospital was considerably higher after TAVR than with standard therapy (699 days [interquartile range, 201 to 720 ] vs. 355 days [interquartile range, 116 to 712 ], $\mathrm{P}<0.001$ ).

\section{Adverse Events}

The rate of stroke was higher in the TAVR group than in the standard-therapy group both at 1 year (11.2\% vs. $5.5 \%, \mathrm{P}=0.06)$ and at 2 years $(13.8$ vs. $5.5 \%, \mathrm{P}=0.01$ ) (Fig. 4 in the Supplementary Appendix). The excess strokes in the TAVR group in the first 30 days were attributable to a greater number of ischemic strokes in that group: there were 1 hemorrhagic and 12 ischemic strokes in the
TAVR group, as compared with 0 hemorrhagic and 3 ischemic strokes in the standard-therapy group ( $\mathrm{P}=0.01)$. Beyond 30 days and up to 2 years, the excess of strokes was attributable to hemorrhagic events: there were 4 hemorrhagic and 5 ischemic strokes in the TAVR group, as compared with 1 hemorrhagic and 4 ischemic strokes in the standard-therapy group. Despite an increase in the rate of stroke, the composite end point at 2 years of the rate of death or stroke was considerably lower in the TAVR group than in the standard-therapy group $(46.1 \%$ vs. $68.0 \%, \mathrm{P}<0.001)$.

The results of other clinical end points are shown in Table 1. Between year 1 and year 2, the frequencies of clinically important complications (including myocardial infarction, major bleeding, renal failure, endocarditis, and the need for new pacemakers) were similar in the TAVR and standard-therapy groups.

\section{Echocardiographic Findings}

Echocardiographic analyses showed that the early hemodynamic benefits of TAVR ${ }^{11}$ were sustained at 2 years (Table 2). Although there was trace or mild paravalvular regurgitation in most of the patients who underwent TAVR, moderate-to-severe paravalvular aortic regurgitation was present in only $10 \%$ of the patients. When both central and paravalvular regurgitation were considered in an evaluation of the total volume load, the TAVR group 


\begin{tabular}{|c|c|c|c|c|c|c|}
\hline \multirow[t]{2}{*}{ Outcome } & \multicolumn{3}{|c|}{1 Year } & \multicolumn{3}{|c|}{2 Years } \\
\hline & $\begin{array}{c}\text { TAVR } \\
(\mathrm{N}=179)\end{array}$ & $\begin{array}{l}\text { Standard } \\
\text { Therapy } \\
(\mathrm{N}=179)\end{array}$ & P Value & $\begin{array}{c}\text { TAVR } \\
(\mathrm{N}=179)\end{array}$ & $\begin{array}{l}\text { Standard } \\
\text { Therapy } \\
(\mathrm{N}=179)\end{array}$ & P Value \\
\hline All-cause mortality — no. (\%) & $55(30.7)$ & $89(50.7)$ & $<0.001$ & $77(43.3)$ & $117(68.0)$ & $<0.001$ \\
\hline Stroke - no. (\%) & $19(11.2)$ & $8(5.5)$ & 0.06 & $22(13.8)$ & $8(5.5)$ & 0.01 \\
\hline Rehospitalization — no. (\%) & $43(27.0)$ & $79(53.9)$ & $<0.001$ & $53(35.0)$ & $95(72.5)$ & $<0.001$ \\
\hline Death or rehospitalization — no. (\%) & $79(44.1)$ & $126(71.6)$ & $<0.001$ & $101(56.7)$ & $153(87.9)$ & $<0.001$ \\
\hline Death or stroke - no. (\%) & $63(35.2)$ & $90(51.3)$ & 0.002 & $82(46.1)$ & $117(68.0)$ & $<0.001$ \\
\hline Cardiac death — no. (\%) & $35(20.5)$ & $75(44.6)$ & $<0.001$ & $50(31.0)$ & $100(62.4)$ & $<0.001$ \\
\hline NYHA class III or IV — no./total no. (\%) & $28 / 118(23.7)$ & $48 / 79(60.8)$ & $<0.001$ & 16/95 (16.8) & $23 / 40(57.5)$ & $<0.001$ \\
\hline Myocardial infarction — no. (\%) & $1(0.8)$ & $1(0.7)$ & 0.91 & $2(1.6)$ & $2(2.5)$ & 0.69 \\
\hline Creatinine $>3 \mathrm{mg} / \mathrm{dl}-$ no. (\%) & $2(1.1)$ & $5(2.8)$ & 0.45 & $2(1.1)$ & $5(2.8)$ & 0.45 \\
\hline Renal failure - no. (\%) $†$ & $4(2.3)$ & $7(4.7)$ & 0.26 & $5(3.2)$ & $9(7.6)$ & 0.15 \\
\hline Major bleeding — no. (\%) & $42(24.2)$ & $21(14.9)$ & 0.04 & $48(28.9)$ & $25(20.1)$ & 0.09 \\
\hline Balloon aortic valvuloplasty — no. (\%) & $2(1.1)$ & $138(82.3)$ & $<0.001$ & $4(2.8)$ & $140(85.3)$ & $<0.001$ \\
\hline Aortic-valve replacement — no. (\%) & 0 & $10(7.6)$ & 0.002 & $1(0.9)$ & $11(8.9)$ & 0.005 \\
\hline Endocarditis - no. (\%) & $2(1.4)$ & $1(0.8)$ & 0.62 & $3(2.3)$ & $1(0.8)$ & 0.32 \\
\hline New pacemaker - no. (\%) & $8(4.7)$ & $14(8.6)$ & 0.15 & $10(6.4)$ & $14(8.6)$ & 0.47 \\
\hline
\end{tabular}

* Percentages shown are Kaplan-Meier estimates, and P values are point-in-time analyses, with the exception of the percentages for creatinine $>3 \mathrm{mg} / \mathrm{dl}$ (265 $\mu \mathrm{mol} /$ liter) and for New York Heart Association (NYHA) class III or IV, which are straight frequencies, with P values calculated with the use of Fisher's exact test. All events in this table were adjudicated by an independent clinical events committee. Disparities with values in our previous report ${ }^{2}$ are due to updated data, based on new reports. TAVR denotes transcatheter aortic-valve replacement. $\uparrow$ Renal failure was defined by the need for dialysis for any length of time.

and the standard-therapy group had similar degrees of total aortic regurgitation at both 1 year and 2 years, owing to the higher prevalence and severity of valvular regurgitation among patients in the standard-therapy group. Among the 61 patients in the TAVR group for whom the results of baseline, year 1 , and year 2 echocardiographic studies were available, paravalvular aortic regurgitation improved in $42.6 \%$, did not change in $41.0 \%$, and worsened in $16.4 \%$. None of the patients in the TAVR group had aortic regurgitation that worsened to a moderate-to-severe level during the follow-up period. Stratification of 2-year mortality according to the degree of post-procedural paravalvular aortic regurgitation revealed a nonsignificant trend toward higher cardiac mortality among patients with moderate-to-severe paravalvular aortic regurgitation than among those with none or only mild paravalvular aortic regurgitation (36.7\% vs. $27.0 \%$ ) (Fig. 5 in the Supplementary Appendix). There was also a trend toward higher all-cause mortality at 1 year associated with moderate or severe paravalvular aortic regurgitation, but this difference disappeared by 2 years.

\section{DISCUSSION}

In this 2-year analysis of the PARTNER trial involving patients with severe aortic stenosis who were considered, according to expert surgical opinion, not to be suitable candidates for surgery, we found that TAVR was associated with the following: a substantial, sustained, and incremental decrease in mortality, with an inverse relationship between the magnitude of the survival benefit and the number and severity of baseline coexisting conditions; a sustained improvement in quality-of-life measures, including NYHA class, the rate of repeat hospitalizations, and the number of days alive and out of the hospital; an increased frequency of early ischemic strokes ( $\leq 30$ days) but little change in the rate of late ischemic strokes ( $>30$ days); and a sus- 


\begin{tabular}{|c|c|c|c|c|}
\hline Outcome & $\begin{array}{c}\text { Before } \\
\text { Procedure } \\
(\mathrm{N}=164)\end{array}$ & $\begin{array}{c}30 \text { Days after } \\
\text { Procedure } \\
(\mathrm{N}=145)\end{array}$ & $\begin{array}{l}2 \text { Years after } \\
\text { Procedure } \\
(\mathrm{N}=67)\end{array}$ & P Value \\
\hline Peak gradient $-\mathrm{mm} \mathrm{Hg}$ & & & & 0.80 \\
\hline Median† & 71.5 & 18.6 & 18.7 & \\
\hline Interquartile range & $55.1-88.4$ & $14.4-23.8$ & $14.9-25.7$ & \\
\hline Mean gradient $-\mathrm{mm} \mathrm{Hg}$ & & & & 0.59 \\
\hline Median $†-$ & 42.7 & 9.3 & 9.7 & \\
\hline Interquartile range & $32.5-52.4$ & $7.1-12.3$ & $7.7-13.3$ & \\
\hline Aortic-valve area $-\mathrm{cm}^{2} \ddagger$ & & & & 0.89 \\
\hline Median $†$ & 0.62 & 1.50 & 1.53 & \\
\hline Interquartile range & $0.52-0.76$ & $1.19-1.80$ & $1.28-1.85$ & \\
\hline Ejection fraction - \% & & & & 0.69 \\
\hline Median & 56.1 & 60.0 & 59.4 & \\
\hline Interquartile range & $46.8-61.6$ & $55.0-65.0$ & $54.6-60.8$ & \\
\hline Paravalvular aortic insufficiency — no./total no. (\%) & & & & 0.001 \\
\hline None & NA & $22 / 144(15.3)$ & $23 / 67(34.3)$ & \\
\hline Trace & & $29 / 144(20.1)$ & $21 / 67(31.3)$ & \\
\hline Mild & & $75 / 144(52.1)$ & $20 / 67(29.9)$ & \\
\hline Moderate & & $17 / 144(11.8)$ & $3 / 67(4.5)$ & \\
\hline Severe & & $1 / 144(0.7)$ & 0 & \\
\hline Transvalvular aortic insufficiency — no./total no. (\%) & & & & 0.75 \\
\hline None & $20 / 164(12.2)$ & $47 / 144(32.6)$ & $19 / 67(28.4)$ & \\
\hline Trace & $35 / 164(21.3)$ & $56 / 144(38.9)$ & $33 / 67(49.3)$ & \\
\hline Mild & $75 / 164(45.7)$ & $40 / 144(27.8)$ & 12/67 (17.9) & \\
\hline Moderate & $30 / 164(18.3)$ & $1 / 144(0.7)$ & $3 / 67(4.5)$ & \\
\hline Severe & $4 / 164(2.4)$ & 0 & 0 & \\
\hline
\end{tabular}

* Total numbers at the three time points are the numbers of patients for whom data on ejection fraction were available at each of those time points. Data for peak gradient and for mean gradient were available for 162 patients before the procedure, 143 patients 30 days after the procedure, and 65 patients 2 years after the procedure; the corresponding numbers for aortic-valve area were 158, 137, and 65. The $P$ values are for the difference between 30 days and 2 years. NA denotes not applicable.

$\uparrow \mathrm{P}<0.001$ for the difference between preprocedural and 30-day values.

The aortic-valve area was calculated with the use of the continuity equation for effective orifice area.

tained improvement in the hemodynamic performance of the valve, with no significant deterioration over time.

The 2-year mortality among patients in the standard-therapy group was striking (68\%); half the patients who had been alive at 1 year died between year 1 and year 2, confirming that the prognosis of symptomatic aortic stenosis is very poor when valve replacement is not performed. It is most likely that this very high mortality is a result not only of untreated aortic stenosis but also of multiple coexisting conditions in this elderly patient population. ${ }^{12-14}$ TAVR was clearly superior to standard therapy in reducing all-cause and cardiac mortality; the number of patients needed to prevent any death and the number needed to prevent a cardiac death in the first 2 years were 4.0 and 3.2, respectively. In a comparison of patients in the two groups who were alive after 1 year, the subsequent mortality at 2 years was $18.2 \%$ in the TAVR group, as compared with $35.1 \%$ in the standard-therapy group (hazard ratio, 0.58; $95 \%$ CI, 0.36 to $0.92 ; \mathrm{P}=0.02$ with the use of the logrank test), which is similar to the data at 1 year 
(hazard ratio, $0.57 ; 95 \% \mathrm{CI}, 0.44$ to $0.75 ; \mathrm{P}<0.001$ with the use of the log-rank test). Hence, the continued divergence between the treatment groups in mortality at year 2 is significant. The findings for cardiac mortality were similar.

Although the magnitude of the reduction in mortality seen after TAVR is striking and unusual for any cardiovascular therapy, there remained a sobering $43 \%$ absolute mortality among patients in the TAVR group at 2 years. Therefore, it would be worthwhile to try to identify an at-risk population that would derive little or no long-term benefit from TAVR owing to irreversible coexisting conditions or other factors. In a subgroup analysis of mortality outcomes according to surgical risk (with the use of the STS score), the mortality benefit with TAVR decreased with increasing STS score (Fig. 2). In contrast, patients in the standardtherapy group had a consistently poor prognosis irrespective of the STS risk stratum. Perhaps some patients with very high STS scores should be viewed as having such severe coexisting conditions that TAVR therapy may be a futile exercise. On the other hand, patients with low STS risk scores $(<5 \%)$ who are not considered to be suitable candidates for surgery, owing largely to anatomical factors (Fig. 6 in the Supplementary Appendix), had the most pronounced mortality reduction with TAVR. Although no definite recommendations can be made about an absolute STS cutoff value until larger patient cohorts are studied, treating physicians should be mindful of the number and severity of coexisting conditions in their patients.

TAVR was also associated with an improvement in quality-of-life measures, including a sustained reduction in cardiac symptoms and fewer repeat hospitalizations, extending into the second year of follow-up. The median number of days alive and out of the hospital was almost twice as high in the TAVR group as in the standard-therapy group. This has substantial implications not only for quality of life, which has been noted by others to improve after TAVR, ${ }^{15-19}$ but also for the cumulative cost of medical treatment associated with rehospitalization, and lends further support to the cost-effectiveness of TAVR as reported thus far. ${ }^{20}$

Stroke is a disturbing complication of TAVR; after 2 years, the frequency of stroke was higher by a factor of 2 with TAVR as compared with standard therapy. This difference appeared to be driven predominantly by embolic ischemic events in the periprocedural period, as shown in other stud- ies. ${ }^{21-23}$ Between 30 days and 2 years after TAVR, the rate of stroke was still higher in the TAVR group than in the standard-therapy group $(2.8 \%$ vs. 5.0\%) (Fig. 4 in the Supplementary Appendix), but the excess was driven primarily by hemorrhagic events. These findings do not support a continued risk of device-related embolic events beyond the first month. Cerebrovascular events in this high-risk patient population are the result of a complex interaction of many factors, including the presence of atherosclerotic aortic and carotid disease, atrial fibrillation, ${ }^{24}$ traumatic head injuries in the elderly (Table 2 in the Supplementary Appendix), and concomitant anticoagulation and antiplatelet therapy. ${ }^{25} \mathrm{~A}$ reduction in the diameter of the device, the use of distal protection devices, ${ }^{26,27}$ and better standardization of antiplatelet and anticoagulation therapy ${ }^{28}$ may be helpful in reducing the incidence of early strokes. Late strokes may represent a constant hazard in this high-risk elderly population.

Echocardiographic analyses revealed excellent valve hemodynamics that remained durable over the course of 2 years, with no evidence of valve migration, leaflet thickening, or calcification. These data on performance of the valves 2 years after TAVR are reassuring because all the echocardiograms were analyzed at an independent core laboratory. A nonsignificant trend toward higher cardiac mortality, but not all-cause mortality, among patients with moderate-to-severe aortic regurgitation persisted at 2 years. This finding differs from results among high-risk patients who are candidates for surgery, in whom moderate-tosevere paravalvular aortic regurgitation is a significant predictor of 2-year mortality, ${ }^{29}$ as shown previously. ${ }^{7,29-31}$ The heavy burden of coexisting conditions in our cohort of patients, who were not suitable candidates for surgery, may have competed with or masked the adverse effects of paravalvular aortic regurgitation.

In summary, in this randomized trial comparing TAVR with standard therapy in patients with severe aortic stenosis who were not suitable candidates for surgery, TAVR reduced the rates of death and of hospitalization, with an improvement in valve hemodynamics and a reduction in symptoms that were sustained for at least 2 years. These results establish TAVR as the standard of care for symptomatic patients with aortic stenosis who do not have reasonable surgical alternatives. The ultimate value of TAVR in such patients will 
depend on several factors: a further reduction in the risk of TAVR-associated complications, especially early stroke; the demonstration of sustained hemodynamic performance of the bioprosthetic valve; and the careful selection of patients who are not candidates for surgery and who do not have extensive coexisting conditions that might overwhelm the benefits of TAVR and render the intervention futile.

Supported by Edwards Lifesciences.

Disclosure forms provided by the authors are available with the full text of this article at NEJM.org.

\section{REFERENCES}

1. Carabello BA, Paulus WJ. Aortic stenosis. Lancet 2009;373:956-66.

2. Leon MB, Smith CR, Mack M, et al. Transcatheter aortic-valve implantation for aortic stenosis in patients who cannot undergo surgery. N Engl J Med 2010;363: 1597-607.

3. Ross J Jr, Braunwald E. Aortic stenosis. Circulation 1968;38:Suppl:61-7.

4. Turina J, Hess O, Sepulcri F, Krayenbuehl HP. Spontaneous course of aortic valve disease. Eur Heart J 1987;8:471-83.

5. Kelly TA, Rothbart RM, Cooper CM, Kaiser DL, Smucker ML, Gibson RS. Comparison of outcome of asymptomatic to symptomatic patients older than 20 years of age with valvular aortic stenosis. Am J Cardiol 1988;61:123-30.

6. Bonow RO, Carabello BA, Chatterjee $\mathrm{K}$, et al. 2008 Focused update incorporated into the ACC/AHA 2006 guidelines for the management of patients with valvular heart disease: a report of the American College of Cardiology/American Heart Association Task Force on Practice Guidelines (Writing Committee to revise the 1998 guidelines for the management of patients with valvular heart disease): endorsed by the Society of Cardiovascular Anesthesiologists, Society for Cardiovascular Angiography and Interventions, and Society of Thoracic Surgeons. J Am Coll Cardiol 2008;52(13):e1-e142.

7. Moat NE, Ludman P, de Belder MA, et al. Long-term outcomes after transcatheter aortic valve implantation in high-risk patients with severe aortic stenosis: the U.K. TAVI (United Kingdom Transcatheter Aortic Valve Implantation) Registry. J Am Coll Cardiol 2011;58:2130-8.

8. Gurvitch R, Wood DA, Tay EL, et al. Transcatheter aortic valve implantation: durability of clinical and hemodynamic outcomes beyond 3 years in a large patient cohort. Circulation 2010;122:131927.

9. Buellesfeld L, Gerckens U, Schuler G, et al. 2-Year follow-up of patients undergoing transcatheter aortic valve implantation using a self-expanding valve prosthesis. J Am Coll Cardiol 2011;57:1650-7.

10. Ussia GP, Barbanti M, Petronio AS, et al. Transcatheter aortic valve implantation: 3-year outcomes of self-expanding CoreValve prosthesis. Eur Heart J 2012 January 12 (Epub ahead of print).

11. Clavel MA, Webb JG, Pibarot P, et al.
Comparison of the hemodynamic performance of percutaneous and surgical bioprostheses for the treatment of severe aortic stenosis. J Am Coll Cardiol 2009; 53:1883-91.

12. Thomas M, Schymik G, Walther T, et al. One-year outcomes of cohort 1 in the Edwards SAPIEN Aortic Bioprosthesis European Outcome (SOURCE) Registry: the European registry of transcatheter aortic valve implantation using the Edwards SAPIEN valve. Circulation 2011;124:42533.

13. Schueler R, Hammerstingl C, Sinning JM, Nickenig G, Omran H. Prognosis of octogenarians with severe aortic valve stenosis at high risk for cardiovascular surgery. Heart 2010;96:1831-6.

14. Wenaweser P, Pilgrim T, Kadner A, et al. Clinical outcomes of patients with severe aortic stenosis at increased surgical risk according to treatment modality. J Am Coll Cardiol 2011;58:2151-62.

15. Watt M, Mealing S, Eaton J, et al. Cost-effectiveness of transcatheter aortic valve replacement in patients ineligible for conventional aortic valve replacement. Heart 2012;98:370-6.

16. Ussia GP, Barbanti M, Cammalleri V, et al. Quality-of-life in elderly patients one year after transcatheter aortic valve implantation for severe aortic stenosis. EuroIntervention 2011;7:573-9.

17. Krane M, Deutsch MA, Bleiziffer S, et al. Quality of life among patients undergoing transcatheter aortic valve implantation. Am Heart J 2010;160:451-7.

18. Reynolds MR, Magnuson EA, Lei Y, et al. Health-related quality of life after transcatheter aortic valve replacement in inoperable patients with severe aortic stenosis. Circulation 2011;124:1964-72.

19. Ussia GP, Mulè $M$, Barbanti $M$, et al. Quality of life assessment after percutaneous aortic valve implantation. Eur Heart J 2009;30:1790-6.

20. Reynolds MR, Magnuson EA, Wang $\mathrm{K}$, et al. Cost-effectiveness of transcatheter aortic valve replacement compared with standard care among inoperable patients with severe aortic stenosis: results from the Placement of Aortic Transcatheter Valves (PARTNER) Trial (Cohort B). Circulation 2012;125:1102-9.

21. Ghanem A, Müller A, Nähle CP, et al. Risk and fate of cerebral embolism after transfemoral aortic valve implantation: a prospective pilot study with diffusionweighted magnetic resonance imaging. J Am Coll Cardiol 2010;55:1427-32.

22. Kahlert P, Knipp SC, Schlamann M, et al. Silent and apparent cerebral ischemia after percutaneous transfemoral aortic valve implantation: a diffusion-weighted magnetic resonance imaging study. Circulation 2010;121:870-8.

23. Tay EL, Gurvitch R, Wijesinghe N, et al. A high-risk period for cerebrovascular events exists after transcatheter aortic valve implantation. JACC Cardiovasc Interv 2011;4:1290-7.

24. Amat-Santos IJ, Rodés-Cabau J, Urena $\mathrm{M}$, et al. Incidence, predictive factors, and prognostic value of new-onset atrial fibrillation following transcatheter aortic valve implantation. J Am Coll Cardiol 2012;59: 178-88.

25. Daneault B, Kirtane AJ, Kodali SK, et al. Stroke associated with surgical and transcatheter treatment of aortic stenosis: a comprehensive review. J Am Coll Cardiol 2011;58:2143-50.

26. Nietlispach F, Wijesinghe N, Gurvitch $\mathrm{R}$, et al. An embolic deflection device for aortic valve interventions. JACC Cardiovasc Interv 2010;3:1133-8.

27. Etienne PY, Papadatos S, Pieters D, et al. Embol-X intraaortic filter and transaortic approach for improved cerebral protection in transcatheter aortic valve implantation. Ann Thorac Surg 2011; 92(5):e95-e96.

28. Ussia GP, Scarabelli M, Mulè M, et al. Dual antiplatelet therapy versus aspirin alone in patients undergoing transcatheter aortic valve implantation. Am J Cardiol 2011;108:1772-6.

29. Kodali SK, Williams MR, Smith CR, et al. Two-year outcomes after transcatheter or surgical aortic-valve replacement. N Engl J Med 2012;366:1686-95.

30. Tamburino C, Capodanno D, Ramondo $\mathrm{A}$, et al. Incidence and predictors of early and late mortality after transcatheter aortic valve implantation in 663 patients with severe aortic stenosis. Circulation 2011;123:299-308.

31. Abdel-Wahab M, Zahn R, Horack M, et al. Aortic regurgitation after transcatheter aortic valve implantation: incidence and early outcome: results from the German transcatheter aortic valve interventions registry. Heart 2011;97:899-906. Copyright (c) 2012 Massachusetts Medical Society. 